\title{
Ulcération chronique dans le cadre d'une atteinte myélomateuse : à propos d'un cas
}

\section{Chronic ulceration in multiple myeloma: report of a case}

\section{RÉSUMÉ}

Une patiente, atteinte d'un myélome multiple, présente une ulcération palatine chronique. Le diagnostic de séquestre osseux en voie d'élimination est retenu.

L'atteinte osseuse, au cours du myélome multiple, est l'une des principales causes de morbidité. La physiopathologie des lésions osseuses est complexe et repose sur le système RANK/RANKL/OPG.

Les bisphosphonates sont utilisées dans la prise en charge des lésions osseuses. Leur principe d'action n'est pas totalement connu mais ils pourraient être à l'origine de nécroses osseuses maxillaires. (Med Buccale Chir Buccale 2006; 12: 15-19).

mots clés : myélome multiple, ulcération, bisphosphonates, ostéolyse

médecine

buccale chirurgie buccale

VOL. $12, \mathrm{~N}^{\circ} 1$ 2006

page 15

\section{SUMMARY}

We report the case of a chronic palatin ulceration in a patient with multiple myeloma. The diagnosis made was osseous sequestra. Bone disease in multiple myeloma is an important cause of morbidity. The physiopathology of bone lesions is complex and based on the interactions between RANK/RANKL/OPG.

Bisphosphonates are used in the treatment of bone lesions, but we still don't know the way they act. They may be responsible of oral necrotic maxillary bone lesions. (Med Buccale Chir Buccale 2006; 12: 15-19).

key words : multiple myeloma, ulceration, bisphosphonates, osteolysis 
Le myélome multiple est une hémopathie maligne caractérisée par la prolifération de lymphocytes $B$ différenciés (plasmocytes). Ce néoplasme, relativement fréquent et commun, représente environ $1 \%$ de tous les cancers et 10 à $15 \%$ des hémopathies malignes. Plus de la moitié des patients ont plus de 60 ans, moins de $3 \%$ des myélomes multiples surviennent avant 40 ans. La fréquence des manifestations bucco-cervicofaciales peut atteindre jusqu'à $70 \%$ dans certaines séries [1]. Dans de rares cas, elles révèlent l'atteinte myélomateuse [2].

Les lésions osseuses ont un aspect caractéristique donnant des images lacunaires «à l'emporte pièce ", sans condensation osseuse périphérique [3]. De nombreuses localisations osseuses ont été rapportées, parfois accompagnées de fractures spontanées. La localisation maxillaire, comme dans le cas présenté, est exceptionnelle [4].

médecine

buccale

chirurgie

buccale

VOL. $12, \mathrm{~N}^{\circ} 1$ 2006

page 16

\section{OBSERVATION}

Une femme de 77 ans consulte pour une ulcération, siégeant dans la région postérieure gauche du palais dur. Selon la patiente, cette ulcération évolue depuis 5 mois. Elle est atteinte depuis 8 ans d'un myélome multiple, à immunoglobuline lgG, avec des chaînes légères Kappa, associé à une protéinurie de Bence Jones. Au cours de l'évolution de la maladie, la patiente bénéficie de divers protocoles thérapeutiques: agent alkylant (Alkéran $囚$ ) et corticoïdes (Solupred $($ )), puis protocole de chimiothérapie de type VAD (vincristine, adriamycine,

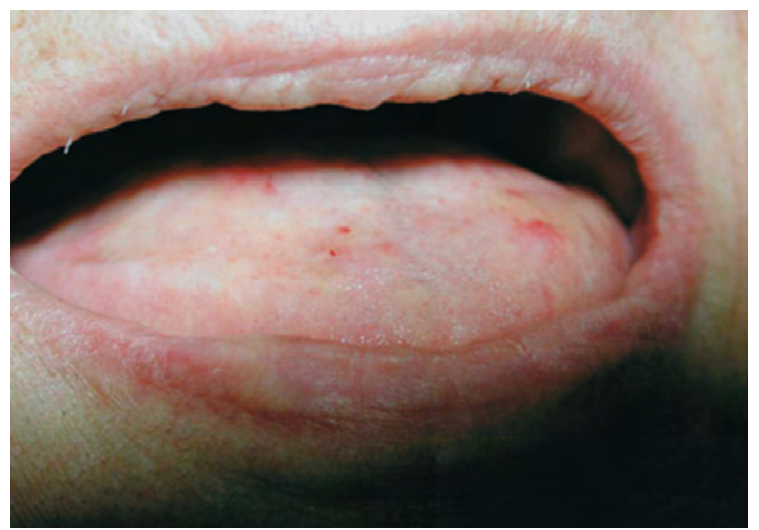

Figure 1 : Face dorsale de la langue. Dorsal side of the tongue
Dectancy|®), pamidronate de sodium et héparines de bas poids moléculaire. Lors de la consultation, le traitement en cours associe thalidomide, hydrocortisone, paracétamol, et anti-vitamines K. La patiente est pâle et asthénique.

L'examen clinique exo-buccal retrouve une hypertrophie bilatérale des glandes submandibulaires secondaire à une fibrose interstitielle. Les régions submandibulaires et sublinguales sont particulièrement indurées, rendant difficile, voire impossible, la recherche d'éventuelles adénopathies cervicales hautes. L'examen endo-buccal révèle une pâleur muqueuse diffuse ainsi qu'une macroglossie majeure due à une amylose. Le dos de la langue est dépapillé et les cicatrices de la glossectomie partielle sont encore visibles. A la palpation, la langue est particulièrement indurée. II est difficile d'examiner la totalité des muqueuses du fait du volume important occupé par la langue (Fig. 1).

Dans la région postérieure gauche du palais dur, il existe une ulcération mesurant environ $1 \mathrm{~cm}$ de diamètre, avec des bords surélevés, rouges et réguliers, avec un fond jaunâtre. Cette lésion est entourée par une zone fortement érythémateuse. La palpation est peu douloureuse ce qui permet de mettre en évidence la dureté de son fond et le caractère acéré de ses bords (Fig. 2). Le diagnostic de séquestre osseux en voie d'élimination est posé. Chez cette patiente de 77 ans, en mauvais

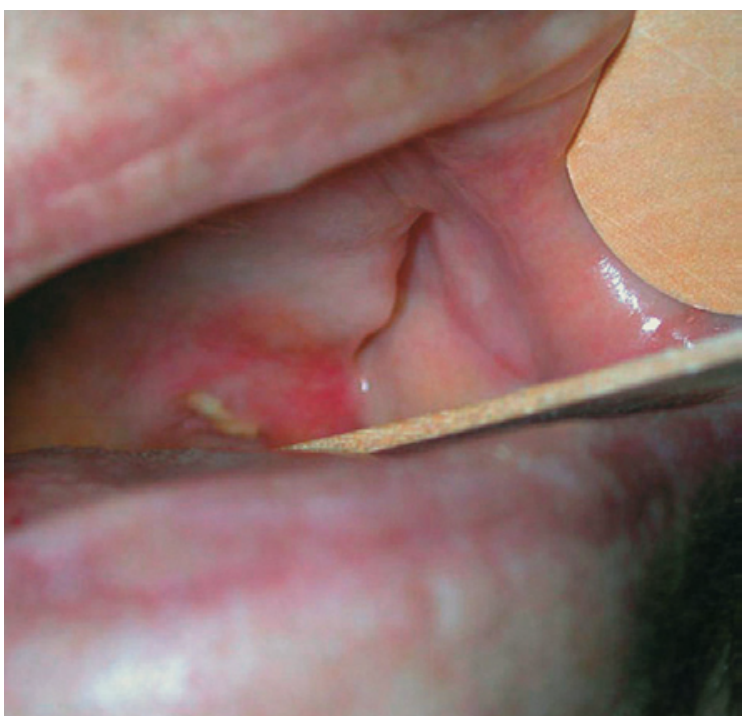

Figure 2 : Ulcération palatine. Palatine ulceration 
état général, n’ayant pas de symptomatologie douloureuse, aucun traitement spécifique n'est mis en route. Une antisepsie locale à base de chlorhexidine (bains de bouche) est recommandée afin de prévenir une surinfection et on préconise de surveiller l'élimination du séquestre osseux.

\section{COMMENTAIRES}

L'espérance de vie des patients atteints d'un myélome multiple n'est pas très longue, puisqu'elle est de 4 ans en moyenne. Pendant ces 4 années, la qualité de vie représente un paramètre important [5]. Les lésions osseuses représentent la manifestation qui affecte sans doute le plus la qualité de la vie; elles constituent donc une cause de morbidité majeure.

Les manifestations radiologiques d'un myélome multiple sont typiquement représentées par de multiples images ostéolytiques avec des limites relativement bien définies, mais sans liseré cortical et sans ostéocondensation périphérique. Elles forment des images «à l'emporte-pièce » qui sont le résultat d'une ostéoclasie sans ostéogenèse compensatrice. Une ostéoporose généralisée

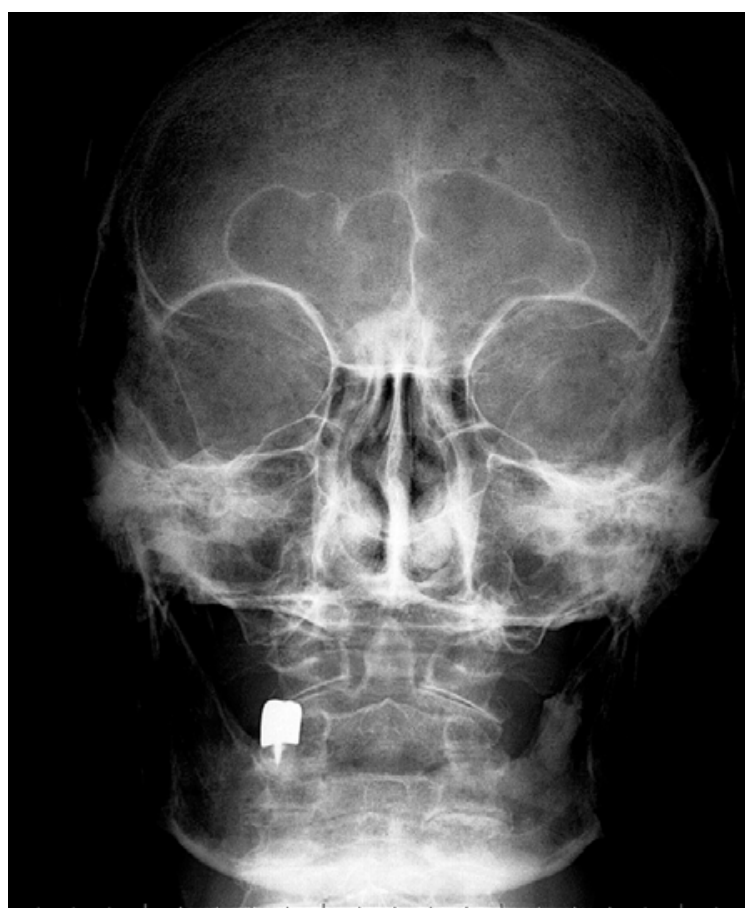

Figure 3 : Radiographie du crâne de face Front cranio-facial $X$-ray peut également être retrouvée [6]. Ces manifestations sont dues à la physiopathologie de l'atteinte myélomateuse elle-même. Dans le cas présenté, la prise d'une corticothérapie au long cours dans un but thérapeutique, l'âge avancé de la patiente et le sexe féminin, sont autant de facteurs qui majorent l'atteinte osseuse (Fig. 3 et 4). Dans le myélome multiple, l'ensemble du squelette osseux peut être atteint, en particulier la colonne vertébrale, le bassin, les côtes, le sternum, les clavicules, l'extrémité supérieure des humérus et des fémurs, ainsi que le crâne [6,7].

Dans la littérature, on retrouve de nombreux cas de manifestations osseuses myélomateuses touchant le maxillaire et surtout la mandibule $[1,2,3,8,9,10,11,12,13]$. Des fractures alvéolaires maxillaires ou mandibulaires, après avulsions dentaires, ont été rapportées. Le fragment osseux forme un séquestre qui s'individualise et s'élimine progressivement $[1,4]$. Dans le cas présenté, il n'y a pas eu d'avulsion récente, pouvant expliquer la formation de ce séquestre. II s'agit probablement d'une fracture spontanée comme on peut en observer, en particulier sur le rachis. Elles ne sont que très rarement rapportées dans la sphère maxillo-faciale. La formation du séquestre osseux,

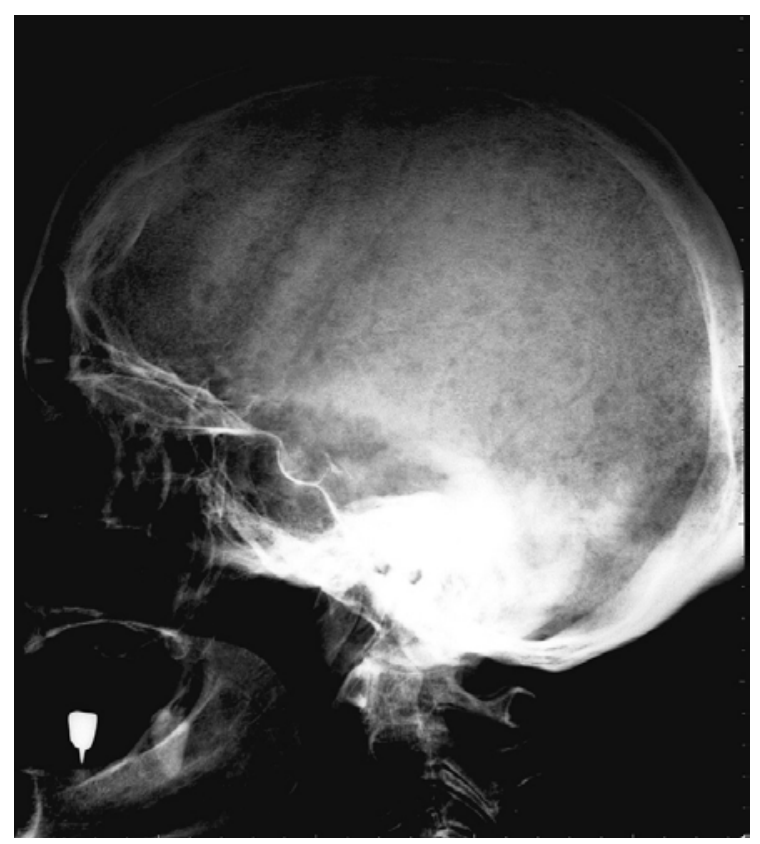

Figure 4 : Radiographie du crâne de profil Side cranio-facial $X$-ray médecine

buccale

chirurgie buccale

VOL. $12, \mathrm{~N}^{\circ} 1$ 2006

page 17 
médecine

buccale

chirurgie

buccale

VOL. $12, \mathrm{~N}^{\circ} 1$ 2006

page 18 chez notre patiente, est inhabituelle de par sa localisation maxillaire et de par son caractère apparemment spontané.

Quelques publications récentes mettent en relation la prise de bisphosphonates avec l'apparition de nécroses osseuses maxillaires ou mandibulaires, chez des patients atteints d'un myélome multiple $[14,15,16]$. La compréhension de la physiopathologie du myélome multiple s'est considérablement améliorée au cours des dernières années. Cependant, les mécanismes d'apparition des lésions osseuses ont été peu étudiés et ils ne sont pas totalement élucidés. Le système RANK (le récepteur activateur du facteur nucléaire kappa B)/RANKL (son ligand)/Ostéoprotégérine (ou OPG) est au centre de la physiopathologie des lésions osseuses [17]. L'interaction RANK/RANKL peut stimuler la résorption du tissu osseux, en augmentant d'une part la formation d'ostéoclastes à partir des précurseurs hématopoïétiques, et d'autre part l'activité même des ostéoclastes.

Dans l'os normal, l'homéostasie dépend de l'équilibre entre la résorption osseuse, déclenchée par l'interaction RANK/RANKL, et l'inhibition de cette résorption par liaison de RANKL avec I'OPG. Cette dernière est une protéine soluble, appartenant également à la famille des TNF (Tumor Necrosis Factor). Elle prévient la résorption osseuse en agissant comme un récepteur «leurre» pour RANKL. Elle diminue la liaison de RANKL avec RANK, donc la différenciation, la multiplication et la fusion des ostéoclastes immatures en ostéoclastes matures [18].

Le rapport entre ostéoclasie et ostéogenèse dépend également d'autres facteurs locaux et systémiques, agissant directement ou par le biais d'autres voies pathologiques, influençant le renouvellement du tissu osseux. Citons les glucocorticoïdes, la vitamine D3, l'hormone parathyroïdienne, les œstrogènes... Ce système a de nombreuses interactions avec les cellules présentes dans la moelle osseuse. Ces interactions permettent aux cellules malignes myélomateuses de déclencher les phénomènes d'ostéolyse. Par ailleurs, les cellules du micro-environnement médullaire jouent un rôle dans la croissance et la survie des cellules myélomateuses, en sécrétant de nombreuses cytokines. La physiopathologie des lésions osseuses est donc un phénomène complexe mettant en jeu de nombreux facteurs cellulaires et chimiques qui entraînent une augmentation de la résorption osseuse, sans ostéogenèse compensatrice [19]. Les cellules malignes myélomateuses stimulent la résorption osseuse de manière directe, soit par la production de facteurs solubles, soit par interaction directe cellule/cellule notamment avec les ostéoclastes, mais également de manière indirecte, en modifiant l'expression de certains facteurs, comme les facteurs de croissance [17].

Depuis quelques années, les bisphosphonates sont considérés comme les molécules de référence dans la prise en charge des lésions osseuses du myélome multiple (régulation de l'ostéolyse, prise en charge de la douleur...). Ils sont à ce titre largement prescrits [19], que ce soit au long cours dans la prise en charge des lésions osseuses ou pour le traitement de l'hypercalcémie maligne. Les dernières publications rapportant des effets secondaires majeurs ophtalmologiques rappellent que leur principe d'action n'est pas encore totalement connu [20]. De même, dans la région bucco-cervico-facial, les bisphosphonates pourraient être à l'origine de nécroses osseuses ischémiques [15]. Des informations complémentaires sont nécessaires avant d'imputer aux bisphosphonates la responsabilité de ces nécroses.

De nouvelles voies de recherche pour la prise en charge des lésions osseuses du myélome multiple sont en voie d'exploration avec un analogue de l'OPG et un antagoniste des $\alpha v \beta 3$ intégrines ayant un potentiel anti-ostéoclasique et antitumoral. Elles ont pour objectif de moduler les effets biochimiques des cellules malignes sur le tissu osseux. Il serait alors possible de maîtriser les atteintes osseuses au cours du myélome multiple et d'améliorer la durée et la qualité de vie des patients. 


\section{RÉFÉRENCES}

1 - Lee SH, Huang JJ, PAN WL, Chan CP. Gingival mass as the primary manifestation of multiple myeloma. Oral Surg Oral Med Oral Pathol Oral Radiol Endod 1996 ; $82: 75-9$.

2 - Mozaffari E, Mupparapu M, OtIs L. Undiagnosed myeloma causing extensive dental bleeding: report of case and review. Oral Surg Oral Med Oral Pathol Oral Radiol Endod $2002 ; 94: 448-53$.

3 - Epstein JB, Voss NJS, SteVEnson-Moore P. Maxillofacial manifestations of multiple myeloma. An unusual case and review of the literature. Oral Surg Oral Med Oral Pathol Oral Radiol Endod 1984 ; 57 : 267-71.

4 - Anacak Y, Kamer S, Donmez A, Haydaroglu A. Destruction of molar roots by metastasis of multiple myeloma. Br J Oral Maxillofac Surg 2002 ; 40 : 515.

5 - Clere D, Fermand JP, Mariette X. Treatment of multiple myeloma. Joint Bone Spine 2003 ; 70 : 175-86.

6 - Croucher PI, ShipMan CM, LIPPITT J, Perry M, Asosingh K, Hijzen A, Brabbs AC, van Beek EJR, Holen I, Skerry TM, Dunstan CR, Russell GR, Van CAMP B, VANDERKERKEN K. Osteoprotegerin inhibits the development of osteolytic bone disease in multiple myeloma. Blood 2001 ; 98 : 3534-40.

7 - Seidl S, Kaufmann H, Drach J. New insights into the physiopathology of multiple myeloma. Lancet Oncol $2003 ; 4$ : 557-64.

8 - VAn Marion AMW, LoKHORst HM, Van Den TweEl JG. Pathology of multiple myeloma. Curr Diagnos Pathol $2003 ; 9: 322-7$.

9 - SIRoIs DA, ChEN SG, GREenBeRG MS. Maxillofacial plasmocytoma resulting in intraoral hemorrhage in a patient with multiple myeloma. Spec Care Dent 1991 ; 11 : 15861.

10 - Kearns GJ, Pogrel MA, Hanks DK, Bruce R. Simultaneous masses of the palate and body of the mandible. J Oral Maxillofac Surg 1993 ; 51 : 413-5.

11 - VINCENT SD, LILLY GE, HUPP JR. Paresthesia of the mandibular division of the trigeminal nerve. J Oral Maxillofac Surg $1993 ; 51: 565-9$.

12 - TAMir R, PICK Al, CALderon S. Plasmacytoma of the mandible: a primary presentation of multiple myeloma. J Oral Maxillofac Surg 1992 ; 50 : 413-5.
13 - Russi E, Odermatt B, Joller-Jemelka HI, Spycher MA. Alveolar haemorrhage as a presenting feature of myeIoma. Europ Resp J 1993 ; 6 : 267-70.

14 - MARX RE. Pamidronate (Aredia) and zoledronate (Zometa) induced avascular necrosis of the jaws: agrowing epidemic. J Oral Maxillofac Surg 2003 ; 61 : 1115-8.

15 - Ruggiero SL, Mehrotra B, Rosenberg TJ, Engroff SL. Osteonecrosis of the jaws associated with the use of bisphosphonates: a review of 63 cases. J Oral Maxillofac Surg 2004 ; 62 : 527-34.

16 - TARASSOFF P, CSERMAK K. Avascular necrosis of the jaws: risk factors in metastatic cancer patients. J Oral Maxillofac Surg 2003 ; 61 : 1238-39.

17 - Croucher PI, Shipman CM, Van Camp B, Vanderkerken K. Bisphosphonates and osteoprotegerin as inhibitors of myeloma bone diseases. Cancer ; 200397 : 81824.

18 - Lacey DL, Timms E, Tan HL, Kelley MJ, Dunstan CR, Burgess T, ELLIOTt R, COlOMBERO A, ELLIOTt G, SCULly S, Hsu H, Sullivan J, Hawkins N, Davy E, Capparelli C, Eli A, Qian YX, Kaufman S, Sarosi I, Shalhoub V, Senaldi G, Guo J, Delaney J, Boyle WJ. Osteoprotegerin ligand is a cytokine that regulates osteoclast differenciation and activation. Cell 1998 ; 93 : 165-76.

19 - KanIS JA, McCloskey EV. Bisphosphonates in multiple myeloma. Cancer 2000 ; 88 : 3022-32.

20 - BISWAS PN, WILTON LV, SHAKIR SAW. Pharmacovigilance study of alendronate in England. Osteoporos Int 2003 ; $14: 507-14$.

NDLR : Dans les quelques mois qui se sont écoulés depuis la soumission de cet article, il y a eu de nombreuses publications concernant l'ostéonécrose maxillaire survenant lors de la prise de bisphosphonates, confirmant la " growing epidemic » prévue par Marx RE. Parmi ces nombreuses publications, on peut signaler la publication en langue française : ABI NAJM S, Lysitsa S, CARREL JP, LESClOUS P, LOMBARDI T, SAMSON J. Bisphosphonates et ostéonécroses des maxillaires. Presse Med 2005 ; 34 : 1073-7.

\begin{tabular}{l}
$\begin{array}{l}\text { médecine } \\
\text { buccale } \\
\text { chirurgie } \\
\text { buccale }\end{array}$ \\
\hline VOL. $12, N^{\circ} 1$ \\
2006 \\
\hline page 19
\end{tabular}

page 19 